\title{
SEMANTIC WEB TECHNOLOGIES MEET BIM FOR ACCESSING AND UNDERSTANDING CULTURAL HERITAGE
}

\author{
E. Iadanza $^{1}$, F. Maietti ${ }^{2}$, A.E. Ziri ${ }^{3}$, R. Di Giulio ${ }^{2}$, M. Medici ${ }^{2}$, F. Ferrari ${ }^{2}$, P. Bonsma ${ }^{4}$, B. Turillazzi ${ }^{1}$ \\ ${ }^{1}$ Consorzio Futuro in Ricerca, Ferrara, Italy, ernesto.iadanza@unifi.it; beatrice.turillazzi@ unibo.it \\ ${ }^{2}$ Università di Ferrara, Dipartimento di Architettura, Ferrara, Italy, (federica.maietti, dgr, marco.medici, federico.ferrari)@unife.it \\ ${ }^{3}$ Nemoris Srl, Bologna, Italy, annaelisabetta.ziri@ nemoris.it \\ ${ }^{4}$ RDF Ltd., Sofia, Bulgaria, peter.bonsma@rdf.bg
}

\section{Commission II}

KEY WORDS: Heritage Documentation, Semantic Web, BIM for Cultural Heritage, Cloud-based Platform, Data Accessing

\begin{abstract}
:
Within the EU funded project INCEPTION - Inclusive Cultural Heritage in Europe through 3D semantic modelling, the keytargeted achievement is the development of a specific cloud based platform, in order to accomplish the main objectives of accessing, understanding and strengthening European Cultural Heritage by means of enriched 3D models. The whole INCEPTION project is based on the close connection between state-of-the-art architectural modeling technologies (BIM, Building Information Modeling) and the latest cutting-edge web technologies. The platform is grounded on semantic web technologies and makes extensive use of WebGL and RESTful APIs, in order to enrich heritage 3D models by using Semantic Web standards. The INCEPTION platform will be a space for interchange of information and for the dialogue among professionals, students, scholars, curators, non-expert users, etc. Furthermore, the Semantic Web structure interlinks the platform with external Cultural Heritage available linked data and makes it gradually enhanced by specific flexible data structures provided as project specific ontologies.

The paper will describe solutions based on the match between BIM, Cloud and Semantic Web.
\end{abstract}

\section{INTRODUCTION}

The ongoing INCEPTION project, which will end in May 2019, aims to inspire and engage more and more people to discover and understand Europe's cultural heritage, and preserve these assets to future generations. To do this, INCEPTION supports Heritage information, ranging from data acquisition, analysis, data management, 3D documentation, new forms of interaction, according to the interdisciplinary approach and to a specific methodology (holistic documentation, optimized data acquisition, data analysis, classification, and interpretation).

Working at heritage architecture and site scale allows the identification of the cultural heritage buildings semantic ontology and data structure for information catalogue. Part of project activities has been addressed to a modelling approach within the 3D semantic Heritage-BIM: the integration of semantic attributes with hierarchically and mutually aggregated 3D geometric models to manage heritage information. 3D parametric and semantic modelling lead to the development of semantic $3 \mathrm{D}$ reconstructions of heritage buildings and sites, integrated with additional documents (i.e. pictures, maps, literature) and intangible information.

\subsection{Overall vision}

At the beginning of the fourth and last year of activity, the INCEPTION project is working on models deployment and widespread sharing, starting from the already developed methodological and technical advancement in 3D data capturing and holistic digital documentation (Di Giulio, 2017).

The overall project workflow is developed starting from requirements (what kind of data, information and visualization issues can be collected and managed by a 3D model according to specific users and needs). In this direction, the integrated data capturing and holistic heritage documentation, the semantic enrichment via 3D modelling in H-BIM environment (Logothetis and Stylianidis, 2016), and the models deployment and enhancement through the INCEPTION platform have been developed.
The H-BIM is the key component making the link between the state-of-the-art of the BIM world and the state-of-the-art of the Cultural Heritage world. H-BIM is a Semantic Web based ontology; this means H-BIM is not software but an open standard. This enables many available tools as well as functionality offered by $\mathrm{W} 3 \mathrm{C}$ and software providers is available for H-BIM allowing INCEPTION to base its solution on proven, mature (where possible open source) tooling.

The ontology H-BIM inherits its BIM part from IFC and ifcOWL where H-BIM was constructed in a way that semantic non-geometrical knowledge and semantic geometrical knowledge is split but interconnected, these clean separations without loose of information is an area where Semantic Web typically excels. This enables handling BIM knowledge while being able to scale up models without performance loss, by cleanly separating data hungry geometry semantics.

The ontology H-BIM inherits its Cultural Heritage knowledge from existing ontologies and state-of-the-art structures / classifications. Both worlds of BIM and $\mathrm{CH}$ are interconnected by adding a layer of mappings as typically applied with Semantic Web technology. The H-BIM is furthermore directly able to be adjusted by third parties extending, improving and adjusting the H-BIM over time and for different aggregations levels; from a single project till country, period or whatever other discriminating aggregation level. This while keeping the core of the INCEPTION Platform working, therefore allowing the INCEPTION platform to be flexible towards different views on how information should be stored according to the H-BIM ontology.

Semantic modelling for Cultural Heritage buildings in BIM environment and the development of the INCEPTION platform for deployment and valorisation of enriched 3D models allow achieving the main purposes of retrieving, knowledge and enhancement of European Cultural Heritage (Vlachidis et al., 2018).

The development of the INCEPTION platform is the keytargeted achievement of the overall project, in order to accomplish the main objectives of accessing, understanding and 
strengthening European cultural heritage by means of enriched 3D models (Maietti et al., 2017).

The platform meets the main aim of realise innovation in 3D models "forever", "for everybody", "from everywhere", by developing, collecting and sharing interoperable 3D semantic models.
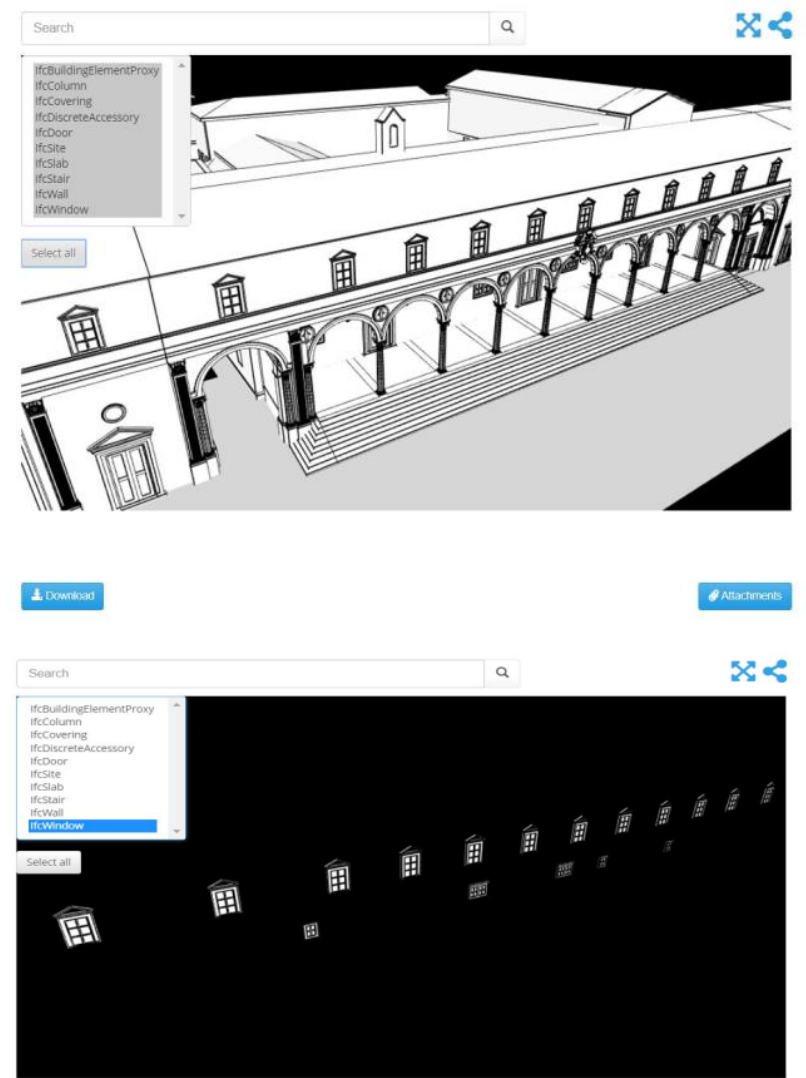

\section{IDomenase}

Figure 1: Above, the BIM model within the INCEPTION platform with all elements selected and displayed. Below, view of one selected element (window)
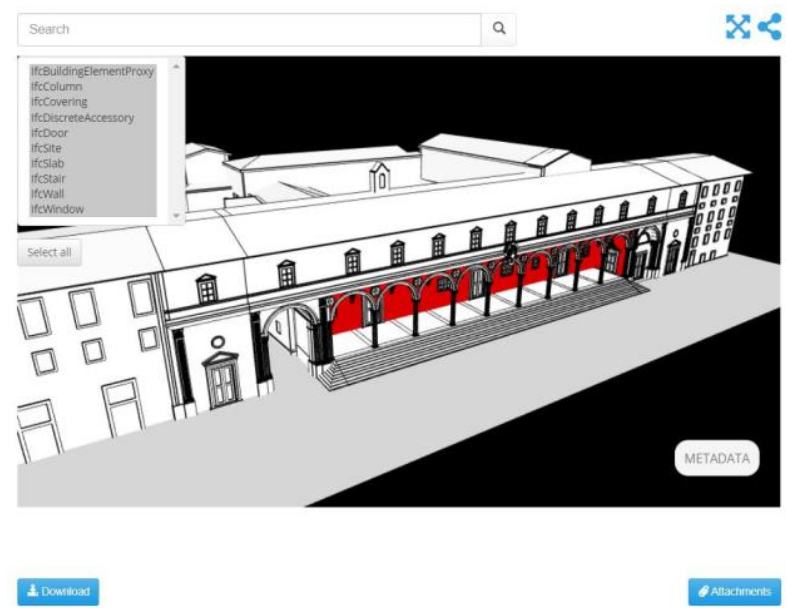

Figure 2: By selecting a specific element of the BIM model, the "Metadata" function is activated
Semantic BIM models uploaded on the platform allow users to interact with the models. Thus, not only "to access" and "to understand" the models, but also to be stimulated "to give the users' perceptual responses to the models" through multilayered information and documentation systems eligible for spatial and multi-criteria queries in a virtual 3D environment (Bustillo et al, 2015).

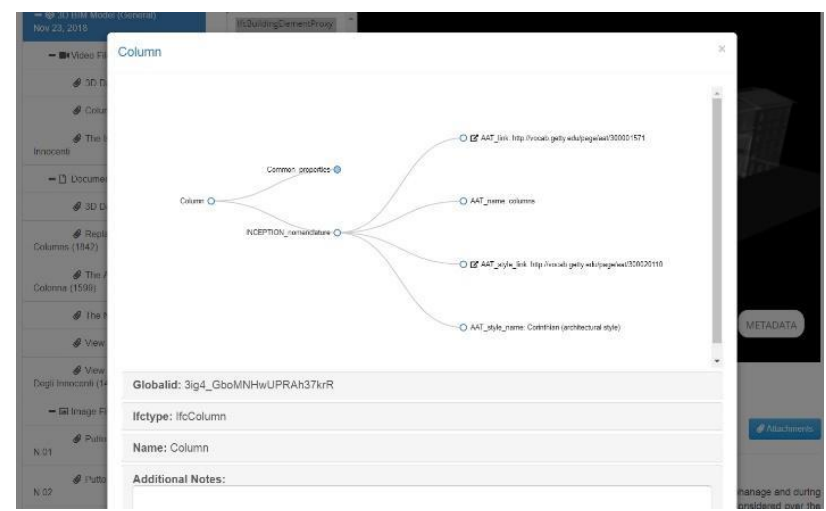

Figure 3: By clicking the function "Metadata", a window opens showing specific information related to the selected element

Therefore, the end-users are able to access information utilising a standard browser, and they are able to query the database using keywords and an easy search method. The search result will display, for instance, a list of H-BIM models, description, historic information and geographic location. INCEPTION open-standard for 3D modelling of cultural heritage (known as "Open H-BIM", an approach based on open standards and workflows) will solve the interoperability issues of data models and associated software.

\section{RELATED WORKS}

INCEPTION is a very specific semantic-based BIM platform for Cultural Heritage sites. Nevertheless, the features included in the INCEPTION semantic BIM platform have been compared to the most known web-based platforms that provide 3D models downloading and exchange. Specific characteristics have been analysed for each platform, in relation to the many design constraints set for the user-experience and technical requirements of the INCEPTION platform (such as enjoyment, navigation, interaction, etc.).

The design verification of the features included in the INCEPTION platform has been compared with the 27 major web-based platforms. The innovative features and application potential of the INCEPTION platform are evident in relation to the major platforms seen as the state of the art.

INCEPTION and all the 27 other platforms have been compared according to some specific indicators:

- Field of Application

- Features of 3D models

- Search functionality

- Visualization Tool

- Accessibility and social

- Download and license

- Quality and access

The assessment has been conducted in order to evaluate the compliance with the main Principles of the London Charter (Denard, 2016): 
- Implementation

- Aims and Methods

- Research Sources

- Documentation

- Documentation Formats and Standards

- Sustainability

- Access

The comparison showed that the platforms related to Cultural Heritage are quite a small number and related to a specific project of documentation / enhancement (Bruschke and Wacker, 2016). They do not count a high number of visits and do not have many $3 \mathrm{D}$ models available. The main reason is that they are linked to specific artefacts related to a museum or they catalogue items in an archaeological site. The quality, however, is high because there is usually a curatorship. The problem (regarding accesses) of these platforms is that their main objective is documentation, cataloguing, and sometimes enhancement.

The most active platforms (in terms of population and visits) are those with a generic vocation and strongly linked to the sharing of 3D models.

The INCEPTION platform works on a more generalist level in the Cultural Heritage field with a strong skill in technical / managerial / maintenance / conservation and development, as well as enhancement through the new possibilities offered by applications such as Augmented and Virtual Reality. The INCEPTION platform will not look like a closed documentation or cataloguing system, but a space for interchange of information (Jessop, 2008), promoting the dialogue between edutainment and the AEC (Architecture Engineering Construction) engineers, between first-class college students, scholars and tour operators (Carrozzino et. al., 2013).

None of the platforms examined is currently implementing semantics about specific content on individual 3D models nor allows (structured or not) access to metadata or paradigms (if any). None of them implements true multi-user access with information, functions, or different data access modes depending on the different types of users. Some of the examined platforms, namely 3D Warehouse, BIM-archiproducts, BIMobject, Library Smartbim, National BIM library and Syncronia, include 3D BIM models management. Just a part of the cited ones includes also a 3D viewer. Most of them include social media interaction, while none of them includes semantic data. Indeed, none of the 27 cited platform includes semantic data management, that is a core functionality for INCEPTION platform. 11 out of 27 provide some semantic search function, but only two (GB3D Type Fossils and Syncronia) can properly manage metadata. All cited platforms are listed in the reference section at the end of the paper.

\section{DEVELOPED METHODOLOGY}

The INCEPTION platform implements semantics about specific contents on individual 3D models and allows access to metadata or paradigms. Moreover, a multi-user access with information, functions, or different data access modes depending on the different typologies of users is allowed (Ramos and Remondino, 2015).

In the cloud-based architecture, the main input is a BIM model of a Heritage site. The models can refer to several categories, such as museums, archaeological sites, historical sites and heritage buildings.

A layered and interoperable ontology has been developed to gather and store new Heritage information into the BIM model matching correct architectural elements to each structural or decorative part of the building. Then the Heritage-BIM $(\mathrm{H}-$ BIM) ontology has been extended to create a connection between external documentation and the whole 3D model or individual elements specified in the H-BIM graph. The Semantic Web technologies, led by the W3C consortium and broadly accepted as the international standard to share machinereadable knowledge content, are the best solution to save and reuse the Heritage documentation related to the building but significant for different domains of interest. The H-BIM ontology has been developed to remap the architectural features from ifcOWL (an open standard for BIM modelling in a semantic web language) and to integrate them with the architectural elements specific of the Cultural Heritage domain, starting from 3D models developed as INCEPTION demonstration cases.

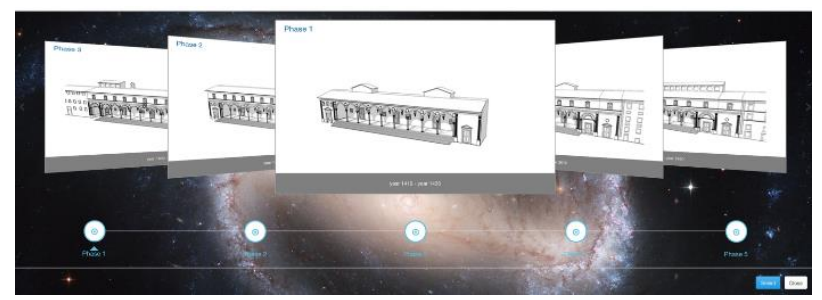

Figure 4: Each element in the BIM model can be provided with a phase it belongs to. This allows the realization of a "time-machine" functionality to work on a specific phase

These models, that embed geometrical and semantic data, are created following a custom and well codified Data Acquisition Protocol, designed during the earlier phases of the INCEPTION Project. All the BIM models are introduced in the platform as IFC (Industry Foundation Class) standard files. The adoption of this standard guarantees that the platform can be accessed regardless of the software used to generate the BIM model.

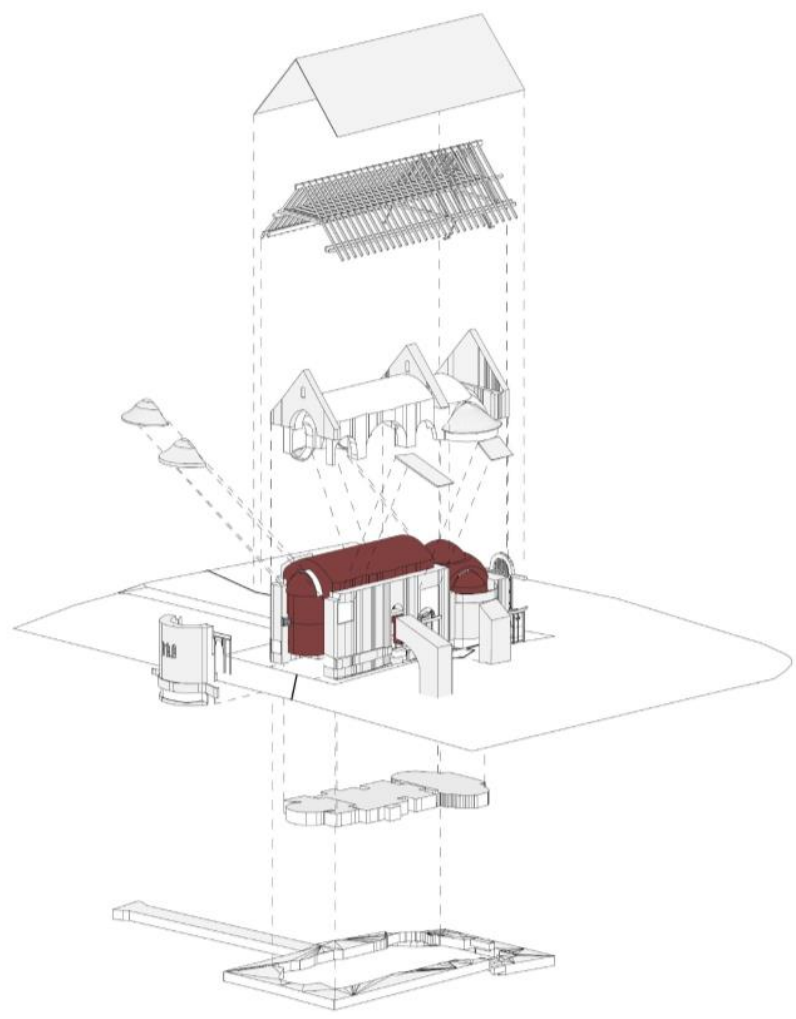

Figure 5: Exploded axonometric view of elements composing the BIM model of the church of Panayia Phorviotissa. Model by

Digital Heritage Lab, Cyprus University of Technology 


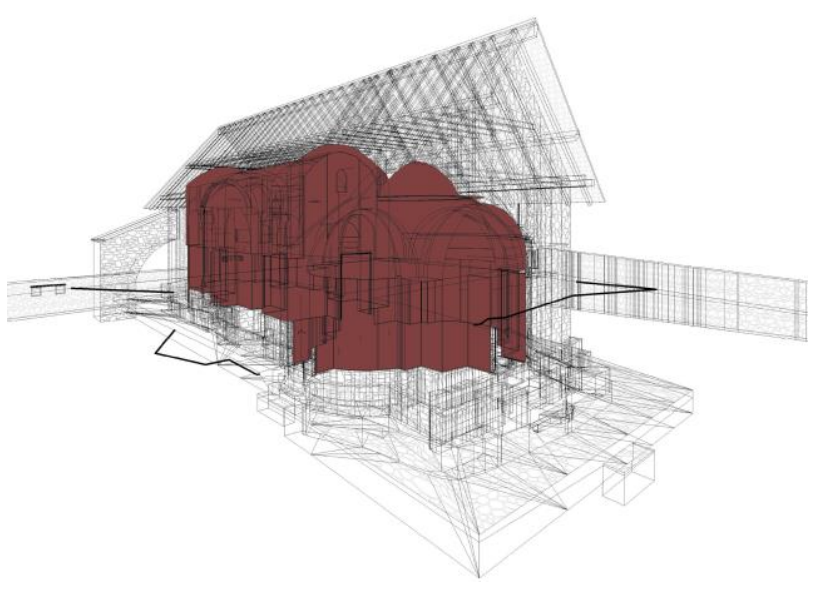

Figure 6: BIM model visualization of the interior frescos of the church of Panayia Phorviotissa in Cyprus
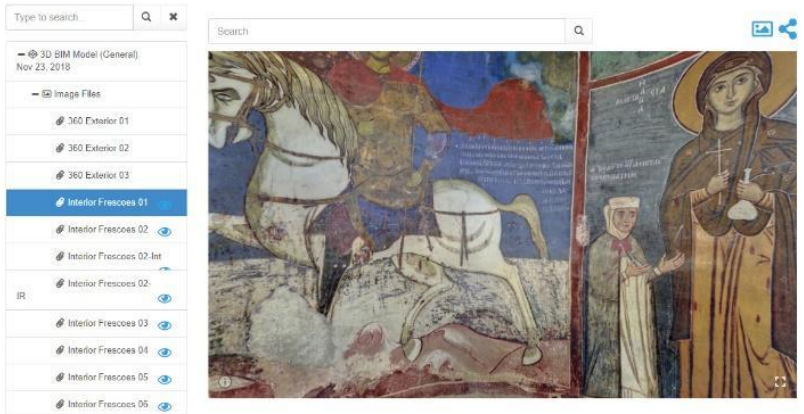

Figure 7: View of the interior frescos of the church of

Panayia Phorviotissa in Cyprus as an attachment linked to the BIM model within the INCEPTION platform

The IFC file is then processed by means of several server-side custom Windows services that extract all the semantic information (both geometries and metadata) and generate Resource Description Framework (RDF) triples, according to the INCEPTION H-BIM ontology, serialized as Turtle (TTL) files. All these triples are stored in a semantic triple store, accessed via HTTP through a dedicated Apache Fuseki SPARQL server. The platform provides the user with the possibility of enriching the models with new semantic metadata (D'Andrea and Fernie, 2013). Indeed, the web client allows to enrich the models with new data (e.g. a date, a value, some textual remarks, etc.) as well as with attachments (e.g. spherical images, thermographic images, 3D models of specific details, videos, etc.), related to the whole $\mathrm{CH}$ site or to a specific geometrical element.

The INCEPTION Platform interacts with its RESTful APIs by means of the SPARQL 1.1 Query Language for RDF, which provides an SQL-like syntax and can be used to query the RDF triple store. From version 1.1 SPARQL offers full CRUD (Create, Read, Update and Delete) functionalities (e.g. SELECT, UPDATE) as well as a useful FILTER expression. It has been constructed to manipulate semantic web triples and data types and aggregations e.g. numbers, strings and URIs. Clearly, the end user does not have to know how to perform a SPARQL query, since the platform interface itself converts graphical requests into queries. In this sense, the query language is transparent to the user.

\subsection{Platform functionalities}

UPLOADs and DOWNLOADs to and from the storage are fully managed by the INCEPTION platform web applications, that integrate functionalities for linking the files to the whole 3D model or to a specific element (e.g. a column or a door), uploading them on a cloud storage, creating the semantic triples to access and link them to the geometries, previewing, downloading, searching etc. The INCEPTION platform webapplications can also act as a stimulus towards third parties to insert the INCEPTION H-BIM standard in their products, by raising awareness to the INCEPTION Platform capabilities.

The above cited cloud storage is dedicated to hosting all the files that complement data for the buildings, such as CAD files, historical documents, images and all the other type of files that could improve the level of information assigned to the object. These files are stored in the INCEPTION cloud storage and managed and organized through dedicated APIs, that also allow users to selectively access this data.

The user will have access to this archive through the INCEPTION platform, by exploiting custom APIs purposely developed for a broad interaction with external components. This open-source object-relational mapping (ORM) system allows thinking to the data model as a set of objects. All the entities created can be exposed as web services, therefore promoting the access to the INCEPTION platform to other solution providers.

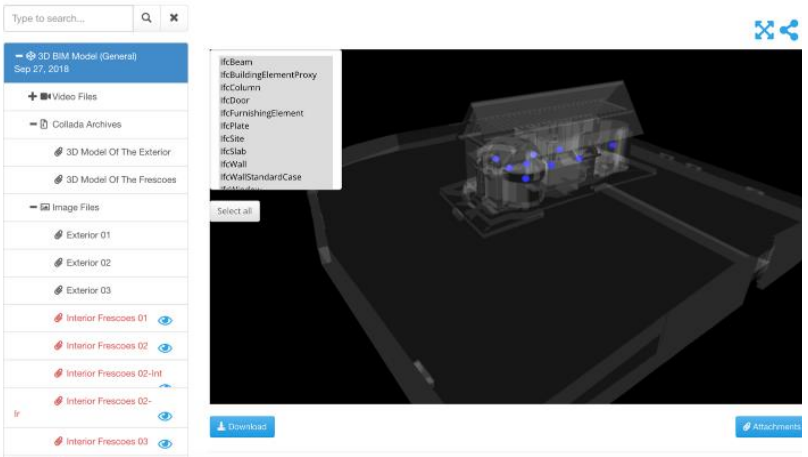

Figure 8: Many files can be linked to specific building elements or placeholders. These links are created as RDF triples and allow the user to always maintain a graphical approach

Moreover, since standard BIM does not completely cover the complex nomenclature of heritage buildings, the ontology classification of building features was integrated with a glossary bridging the gaps in current BIM definitions creating a first hierarchical classification on Heritage buildings. The INCEPTION approach in the selection of the nomenclature examined classical architectural sources: a "taxonomy of monuments" according to holistic e-documentation needs is the base to understand the approach on "names". This was the starting point of a classification in Semantic Web language in order to connect terms with H-BIM ontology. Nevertheless, the glossary was set by a top-down approach, starting from the INCEPTION demonstration cases needs and checking the nomenclature with the Getty AAT, one of the most comprehensive sources of architectural definitions.

The INCEPTION platform exploits the concept of the semantic web. Many web APIs have been developed to provide the INCEPTION H-BIM Interoperable Platform with a REST interface to access the BIM models allowing users to operate on $3 \mathrm{D}$ information as well as on its related semantics. 
This core functionality is dedicated to the end-users as well as to developers and solution providers for interacting with external mobile devices and applications. Mobile phone applications, to be used on site, will access the INCEPTION HBIM Interoperable Platform through this REST web-service, stimulating a flowering of web based mobile applications from third parties. The web application, core of the whole platform, allows users to search 3D models using specific keywords contained in the semantic information.

The platform will be placed on a generalist level in the Cultural Heritage $(\mathrm{CH})$ focusing on buildings and sites, with strong features in technical/managerial/maintenance/conservation and development, as well as enhancement through the new possibilities offered by the Augmented and Virtual reality.

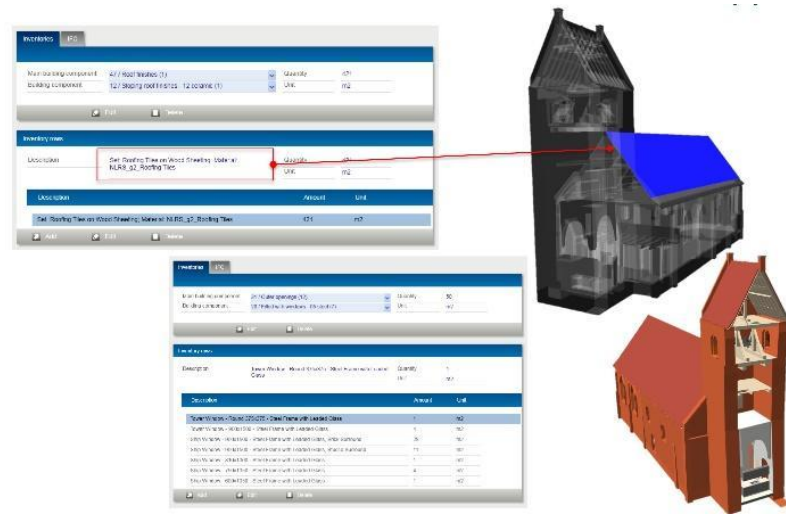

Figure 9: View of the asset management tool linked to the INCEPTION platform. One of the main functionalities is the BIM inventorying and the setting up of different parameters for inspections and the planning of maintenance / conservation strategies

\subsection{H-BIM ontology}

The choice to use ontologies in a BIM environment is challenging. BIM models of heritage buildings are rich of specific features and related data: modelling this domain through Semantic Web technologies could seem a daunting task.

The main outcome of building an H-BIM ontology for the INCEPTION project is the capability to create, exchange and reuse information.

The ontology is an interoperable layer that can be linked with external open data or other ontologies. For instance, external data connected to the ontology are the Getty AAT nomenclature.

The Getty vocabularies (AAT - Art \& Architecture Thesaurus, TGN - Getty Thesaurus of Geographic Names, and ULAN Union List of Artist Names) contain structured terminology for art, architecture, decorative arts, archival materials, visual surrogates, conservation, and bibliographic materials.

Compliant with international IT standards, they provide authoritative information for cataloguers, researchers, and data providers. The publishing of Getty vocabularies is based on SKOS (Simple Knowledge Organization System) allowing a great accessibility and re-usability of the contents. Indeed, more vocabularies could be connected to INCEPTION in the future, without the need for several and significant changes to the structure.

Other projects' ontologies (such as CIDOC CRM, for museums artefacts) can be linked; the INCEPTION ontology can be a flexible way to represent the connection among the different parts of the buildings with external data, like media files or maintenance information.

Another appropriate reason to create an H-BIM ontology is the possibility to open the project data to include them into Europeans Semantic compatible platform like EUROPEANA, without losing information or using specifically created formats. The H-BIM core ontology remaps, as a starting point, some main elements of IfcOWL ontology but simplifies the relations because its scope is not to fully describe the model geometry.

The geometry is stored in the platform, but the main and primary scope of INCEPTION was not to create a full description of mathematical data with Semantic Web languages but to exploit those languages to enrich the model with an interoperable interface.

Using OWL language and a SPARQL endpoint, it was easy in the development of the platform to fully use the power of the data structure to create a complete multidimensional view of the historical site. For instance, INCEPTION adds the fourth dimension to the building models, metadata and external enrichment, using the W3C OWL-Time ontology to describe model phases though time and represent the building evolution.

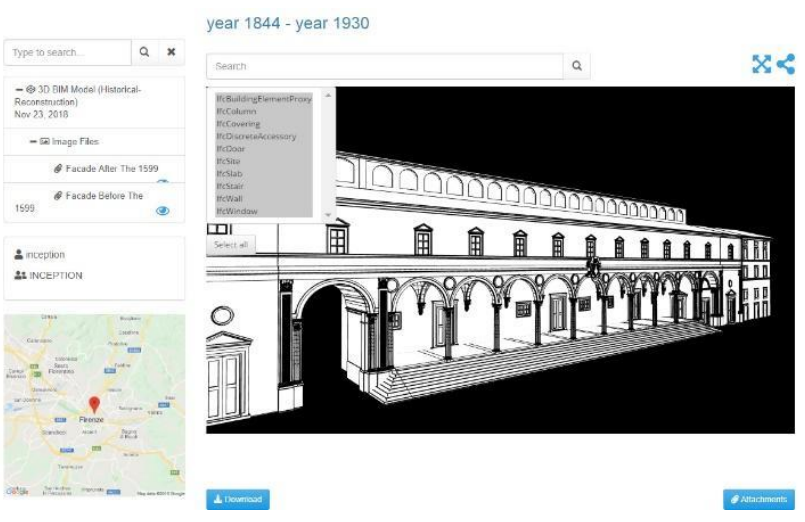

Figure 10: The Time-machine functionality within the

INCEPTION platform. View of the model as it was in the time frame between 1844 and 1930

Another point of view of this specific domain is that historical sites can be modelled with very different aims, from touristic purposes up to the analysis and understanding of aesthetic, historical and cultural significances. This is a particularly relevant point, since the project aim is to increase knowledge, enhancement and dissemination through 3D digital models in order to promote the inclusiveness and accessibility of European assets.

In this direction, several users with different skills in the field of Cultural Heritage have been involved leading the research toward effective strategies to increase use and reuse of digital models. These strategies are aimed at maximizing the impact of using digital data for cultural heritage applications involving a wide range of non-expert and expert users, starting from specific requirements for processing, managing, delivering cultural heritage information to a broad audience.

This complexity of the cultural domain is well handled by the Semantic Web technologies: the INCEPTION ontology uses the triple store to filter the information by time frames, scope or significance, to correctly inform the user accordingly to his skills and purposes.

Thus, managing different kind of data and retrieving them according to the correct time and user typology allows a deeper granularity in the model navigation. 


\section{APPLICATION FIELD}

The INCEPTION overall workflow and the platform functionalities have been developed and tested on a set of selected Demonstration Cases, intended as a cultural heritage asset, which serves as a case study in order to illustrate the applicability of the developed methodologies and tools in a variety of real scenarios. In this direction, a Stakeholder Panel with different skills in the field of Cultural Heritage has been involved starting from the very beginning of project development, in order to lead the research toward effective strategies to increase use and reuse of digital models.

Demonstration Cases under development within INCEPTION are:

Historical and Archaeological Sites

- Old stone villages in Dalmatia, Croatia

- Castillo de Torrelobatón, Valladolid, Spain

- The Acropolis of Erimokastro, Rhodes, Greece

Heritage Sites

- Istituto degli Innocenti, Florence, Italy

- Villa Klonaridi, Athens, Greece

- Saint Nicholas Chapelin, Obergum, The Netherlands

- Church of Panayia (The Virgin) Phorviotissa (Asinou) Nikitari village, Troodos, Cyprus

Museums

- Historical Archives - Museum of Hydra (HAMH), Greece

- Technical Museum Nicola Tesla, Zagreb, Croatia

The selected Demonstration Cases are representative of different kinds and types of Cultural Heritage (archaeological sites, monumental complexes, historical architectural buildings, museums etc.), different states of preservation and differentiated environmental impacts. Furthermore, each Cultural Heritage asset selected is characterized by a different risk factor. Moreover, the selected Demonstration Cases are a "mean of validation" of the selected use cases, A "use case" can be defined as a list of actions, typically defining the interactions between a user and a system, to achieve a goal. Use cases in Cultural Heritage represent the actions defined within the framework of knowledge, understanding, conservation, protection, enhancement, etc. of cultural heritage assets. Use cases are commonly used to define various information (such as architectural, structural etc.) that captures requirements for different applications. They are an easy way to define, describe and analyse user needs and system/methodology capabilities. In addition, use cases fill the gap between user needs and information design, aiding the management of the system complexity. The use cases clearly demonstrate the analytical potential of the new model for research, interpretation, scholarship and innovation in curation and dissemination. Potential use cases include reconstruction, preservation and conservation of Cultural Heritage buildings and sites, such as historic buildings, historical religious churches and sites, monuments with great architectural and artistic value, and in general historical assets. They also address the various interests, expectations and level of information of the three user categories, as developed in accordance to the Stakeholders' requirements.

The first prototype of the INCEPTION platform has been developed based on the macro-needs analysis carried out in the first phase involving users and Stakeholders and developed through the definition of invariants and technological and system constraints. According to Human Cantered Design's principles (Kankainen et. al., 2012), an iterative process has been applied to the first prototype in order to evaluate and implement it together with users and Stakeholders for new versions' development.

The objective was not only to gain opinions on the current state of development of the INCEPTION platform by users, but also and above all to participate with them in the experimentation and re-elaboration of the opportunities offered by the platform as defined through the first analyses.

The aim of this activity was to understand if the first prototype allowed the understanding of the tool, if this understanding allowed a greater awareness on the needs focused during the first analysis, and if this awareness helped to reconsider the structure of the current prototype, improving it.

The purpose was not only to analyse the requests by users and Stakeholders, but also to work together to the design of a new set-up of the platform, responding to needs not yet identified, easy to navigate and interesting for each type user, common visitors or specialized technicians (F. Maietti et. al., 2018).
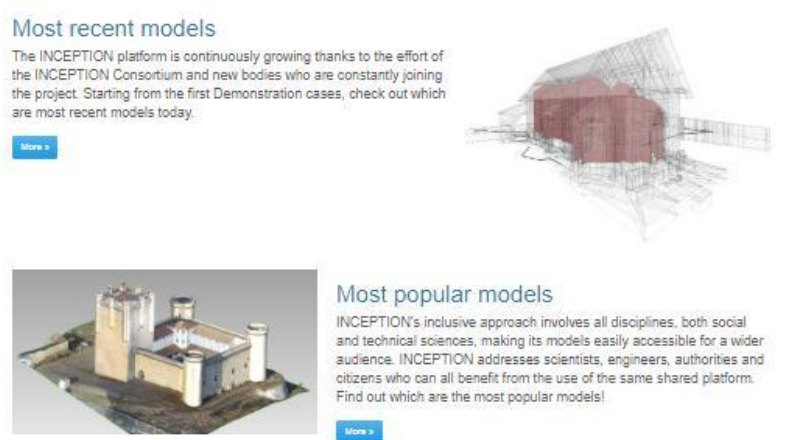

Most popular models INCEPTION sinclusive approach irvolves all discopines, both social INCEPTIONs inclusive approach involves all disciplines, both social
and technical sciences, making its models easily accessible for a wider

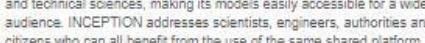
citzens who can all beneff trom the use of the
Find out which are the most popular models! Find out
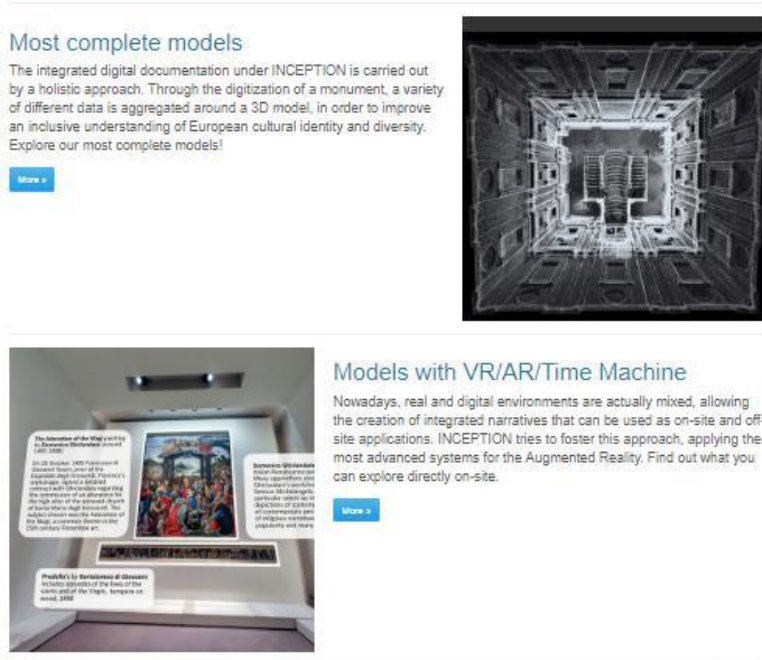

Models with VR/AR/Time Machine Nowadays, real and digital environments are actually mixed, allowing

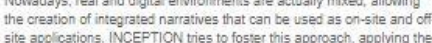
site appications. INCEPTION ties to foster this approash, applying the
most advanced systems for the Augmented Reality. Find out what you
can expore directly on-sige can excolode

Figure 11: The platform, at the current stage of development, allows browsing $3 \mathrm{D}$ models by different users according to different needs and requirements

\section{CONCLUSIONS}

The INCEPTION project, that is going to be completed within the next six months, has defined the approach and the methodology for semantic organization and data management toward H-BIM modelling, and the nomenclature for semantic enrichment of heritage 3D models. The organization of consolidated knowledge is performed following a specific workflow in order to get them suitable for their reuse into $\mathrm{H}$ - 
BIM semantic model, accordingly to digital documentation and capturing protocols that have been developed.

According to the INCEPTION workflow, the H-BIM process starts documenting user needs, including and engaging not only experts but also non-experts. The demand has been leading us to "how" and "what" surveying information to be included in $\mathrm{H}$ BIM. The surveying procedure produces a variety of different data, formats and outputs. It is essential to process that data without losing important information like metadata and paradata while editing and developing the digital elements of the H-BIM. A methodology of archiving digital data and linking them to the final product is one of the main outcomes. Before and during the creation of H-BIM, the nomenclature (vocabularies, thesaurus, etc.) is critical to maintain a common typology and to support interoperability.

Starting from the standardization for H-BIM modelling, the methodology for merging IFC models and semantic data has been defined. The development of a semantic 3D reconstructions, integrated with intangible information and social environment, structuring digital representation of buildings and sites leads to the creation of models more accessible and implementable in a Heritage-BIM environment, based on Open BIM standard (IFC, IFD, etc.).

The INCEPTION platform contains, visualizes, manages, updates, and exchanges technical and popular information regarding historical heritage, through the use of 3D BIM models. Any form of digital or digitized content, stored or linked into a 3D H-BIM model, as well as semantically indexed by the use of the INCEPTION ontology, will allow the use of different navigation systems.

One major challenge within the INCEPTION project is to create support for geometric representations using Semantic Web technology, taking into account the fact that in the area of Cultural Heritage geometry is often derived from point clouds coming from different 3D surveying techniques, and representing this information is therefore often even more space demanding than normal BIM models. Within INCEPTION, it was decided not to escape the issue itself but to look for a solution where these data can really be converted towards content complying with Semantic Web technology. The distinction between geometric and non-geometric semantics as well as the structured setup to still keep all relevant knowledge allows a system in which data-heavy parts can be excluded from the core data stores, resulting in a minimal performance loss, even when handling vast amounts of data (Bonsma et. al., 2018).

The representation of the model and its related holistic enrichment is not limited to simple 3D mapping but takes also into account the "time" dimension, using a W3C interoperable standard to navigate the H-BIM through history. This enhancement has been possible thanks to the choice of open Semantic Web technologies to define the model components.

The next steps, until the conclusion of the project - in May 2019 - will be focused on the acomplishment of the Heritage workflows related to the Demonstration Cases and the further optimization of the platform. This improvement will be related not only on technical features and the platform interface, but also on platform population, by uploading as many $3 \mathrm{D}$ models as possible.

\section{ACKNOWLEDGEMENTS}

The project is under development by a consortium of fourteen partners from ten European countries led by the Department of Architecture of the University of Ferrara. Academic partners of the Consortium, in addition to the Department of Architecture of the University of Ferrara, include the University of Ljubljana (Slovenia), the National Technical University of Athens (Greece), the Cyprus University of Technology (Cyprus), the University of Zagreb (Croatia), the research centers Consorzio Futuro in Ricerca (Italy) and Cartif (Spain).

The clustering of small medium enterprises includes: DEMO Consultants BV (The Netherlands), 3L Architects (Germany), Nemoris (Italy), RDF (Bulgaria), 13BIS Consulting (France), Z $+\mathrm{F}$ (Germany), Vision and Business Consultants (Greece).

The INCEPTION project has been applied under the Work Programme Europe in a changing world - inclusive, innovative and reflective Societies (Call - Reflective Societies: Cultural Heritage and European Identities, Reflective-7-2014, Advanced 3D modelling for accessing and understanding European cultural assets).

This research project has received funding from the European Union's H2020 Framework Programme for research and innovation under Grant agreement no 665220.

\section{REFERENCES}

3dmodelfree.com, 2018. Free 3D Models Download, http://www.3dmodelfree.com (Last accessed on December 2018).

3dsky.org, 2018. 3D models download, http://3dsky.org (Last accessed on December 2018).

3D Warehouse, 2018. http://3dwarehouse.sketchup.com (Last accessed on December 2018).

Archive3d.net, 2018. Free 3D Models and Objects Archive. Download: 3ds, obj, gsm, max models. Available at: http://archive3d.net (Last accessed on December 2018).

Autodesk Online Gallery, 2018. Free 3D models, Rendering images and CAD files, http://gallery.autodesk.com (Last accessed on December 2018).

BIM Archiproducts, 2018. The largest BIM and CAD database for architects and designers. Available at: http://bim.archiproducts.com (Last accessed on December 2018).

BIMobject, 2018. Download free BIM content from the BIMobject Cloud. Available at: http://bimobject.com (Last accessed on December 2018).

Bonsma, P., Bonsma, I., Ziri, A.E., Iadanza, E., Maietti, F., Medici, M., Ferrari, F., Sebastian, R., Bruinenberg, S., Lerones, P.M., 2018. Handling huge and complex 3D geometries with Semantic Web technology. In: Florence Heri-Tech - The Future of Heritage Science and Technologies, IOP Conf. Series: Materials Science and Engineering 364 (2018) 012041.

Blend Swap, 2018. Available at: http://www.blendswap.com (Last accessed on December 2018).

Bruschke, J., Wacker, M., 2016. Simplifying documentation of digital reconstruction processes. In: $3 D$ Research Challenges in Cultural Heritage II. Springer, Cham. pp. 256-271.

Bustillo, A., Alaguero, M., Miguel, I., Saiz, J. M., Iglesias, L. S., 2015. A flexible platform for the creation of 3D semiimmersive environments to teach cultural heritage. Digital Applications in Archaeology and Cultural Heritage, 2(4), pp. 248-259. 
CGTrader, 2018. Available at: http://www.cgtrader.com (Last accessed on December 2018).

Clara.io, 2018. Online 3D Modeling, 3D Rendering, Free 3D Models, http://clara.io (Last accessed on December 2018).

CyArk, 2018. Available at: http://www.cyark.org (Last accessed on December 2018).

Carrozzino, M., Bruno, N., Bergamasco, M., 2013. Designing interaction metaphors for Web3D cultural dissemination. Journal of Cultural Heritage, 14(2), 146-155.

D'Andrea, A., Fernie, K., 2013. CARARE 2.0: a metadata schema for 3D Cultural Objects. In: Digital Heritage International Congress (DigitalHeritage), IEEE Vol. 2, 2013, pp. 137-143.

Denard, H., 2016. A new introduction to the London Charter. In: Paradata and transparency in virtual heritage, Routledge, pp. 83-98.

Digital Designs for Physical Objects, 2018. Available at: http://www.thingiverse.com (Last accessed on December 2018).

Di Giulio, R., Maietti, F., Piaia, E., Medici, M., Ferrari, F., \& Turillazzi, B., 2017. Integrated data capturing requirements for 3D semantic modelling of cultural heritage: the INCEPTION protocol. International Archives of the Photogrammetry, Remote Sensing and Spatial Information Sciences, 42(2/W3), pp. 251-257.

GB3D Type Fossils, 2018. High-resolution photographs and digital models of British type fossils. Available at: http://www.3d-fossils.ac.uk (Last accessed on December 2018).

Jessop, M., 2008. Digital visualization as a scholarly activity. Literary and Linguistic Computing, 23(3), 281-293.

Kankainen, A., Vaajakallio, K., Kantola, V., Mattelmaki, T., 2012. Storytelling Group - A co-design method for service design. Behaviour \& In-formation Technology, Volume 31, Issue 3, pp. 221-230.

Library Smartbim, 2018. Free Parametric, Data-Rich Revit Objects. Available at: http://library.smartbim.com (Last accessed on December 2018).

Logothetis, S., Stylianidis, E., 2016. BIM Open Source Software (OSS) for the documentation of cultural heritage. Virtual Archaeology Review, 7(15), pp. 28-35.

Maietti, F., Di Giulio, R., Balzani, M., Piaia, E., Medici, M., Ferrari, F., 2017. Digital Memory and Integrated Data Capturing: Innovations for an Inclusive Cultural Heritage in Europe through 3D Semantic Modelling. In Mixed Reality and Gamification for Cultural Heritage. Springer, Cham, pp. 225244.

Maietti, F., Piaia, E., Mincolelli, G., Di Giulio, R., Imbesi, S., Marchi, M., Giacobone, G.A., Brunoro, S., 2018. Accessing and understanding Cultural Heritage through users experience within the INCEPTION project. In: Digital Heritage Progress in Cultural Heritage: Documentation, Preservation, and Protection, Springer, Cham. pp. 356-365.

MyMiniFactory.com, 2018. https://www.myminifactory.com (Last accessed on December 2018).

NBS National BIM Library, 2018. Free to download BIM objects. Available at: http://www.nationalbimlibrary.com (Last accessed on December 2018).
Ornament 3D, 2018. Available at: http://ornament3d.org (Last accessed on December 2018).

Ramos, M. M., Remondino, F., 2015. Data fusion in cultural heritage a review. ISPRS-Int. Archives of the Photogrammetry, Remote Sensing and Spatial Information Sciences, 5, W7, pp. 359-363.

Smithsonian 3D digitization, 2018. Available at: http://3d.si.edu (Last accessed on December 2018).

Sketchfab, 2018. 3D content on web, mobile, AR, and VR. Available at: http://sketchfab.com (Last accessed on December 2018).

Syncronia.com, 2018. http://www.syncronia.com (Last accessed on December 2018).

Three D Scans, 2018. Available at: http://threedscans.com (Last accessed on December 2018).

TurboSquid, 2018. 3D Models for Professionals. Available at: http://www.turbosquid.com (Last accessed on December 2018).

Unity Asset Store, 2018. Available at: http://www.assetstore.unity3d.com (Last accessed on December 2018).

Vlachidis, A., Bikakis, A., Kyriaki-Manessi, D., Triantafyllou, I., Padfield, J., Kontiza, K., 2018. Semantic representation and enrichment of cultural heritage information for fostering reinterpretation and reflection on the European history. In Digital Cultural Heritage. Springer, Cham, pp. 91-103.

W3C. World Wide Web Consortium, 2018 Available at: https://www.w3.org/ (Last accessed on December 2018).OWLTime Ontology, W3C of October 2017. Avalaible at: https://www.w3.org/TR/owl-time/ (Last accessed on December 2018).

Zamani Project, 2018. Available at: http://zamaniproject.org (Last accessed on December 2018). 